Adversity, attachment, and mentalizing

\title{
Adversity, attachment, and mentalizing
}

\section{Peter Fonagy, PhD, FBA, FMedSci ${ }^{\mathrm{a}, \mathrm{b}, *}$ and Anthony W. Bateman, MA, FRCPsych ${ }^{\mathrm{a}, \mathrm{b}}$}

\author{
a Research Department of Clinical, Educational and Health Psychology, University College \\ London, 1-19 Torrington Place, London WC1E 7HB, UK \\ ${ }^{\mathrm{b}}$ The Anna Freud Centre, 12 Maresfield Gardens, London NW3 5SU, UK \\ * Corresponding author. E-mail: p.fonagy@ucl.ac.uk
}

\begin{abstract}
The papers in this special issue offer evidence of personality disorder as a dysfunction of higher-order cognition, which is conceptualized variously as a disorder of mentalizing, metacognition, mindfulness, social cognition and reflective function. While there may be differences in the scope of these concepts, they all imply that higher-order mental processing is at the core of personality function. In this commentary, the authors use mentalizing as an umbrella term for these concepts, and argue that it is the complex interaction of adversity, attachment and mentalizing that lead to the characteristic symptoms of borderline personality disorder and other personality disorders. Evidence is provided from the papers in this special issue, comments made on the findings and further avenues for research are recommended.
\end{abstract}

Keywords: Mentalizing, metacognition, mindfulness, reflective function, higher-order cognition, borderline personality disorder, adversity, attachment. 
Adversity, attachment, and mentalizing

\section{Introduction}

The papers in this special issue are connected by a shared aim of creating connections between our understanding of personality disorder and its social causes via the study of higher-order cognitive processes. Higher-order cognition (HOC) refers to informationprocessing mechanisms that do not rely on a static, fixed set of specialised brain regions and fixed neuroanatomical connections [1]. HOC is thought to operate by optimizing neural resources and creating routes between processing systems. Normally, neural networks are believed to manifest collective behaviours, which become apparent in second- or higherorder parameters coming to govern contextual information processing modes [2]. Whether we describe HOC as reflective functioning (RF) [3], mentalizing [4], social cognition [5], metacognition [6,7] or mindfulness [8], the core distinguishing feature of these hypothetical constructs is that they relate to brain structure as a hierarchy of layers of abstraction and assume a top-down influence on lower orders of this neural pyramid.

All the above concepts assume that a process, something like self-awareness, can dynamically control local biophysical and biochemical parameters to facilitate access to particular processing units, which have physical locations, and simplify the control of the complex device that is the brain, functioning as an information-processing system. In this type of conceptualization we assume that the brain functions as some kind of interpreter of code and the higher-order constructs such as metacognition occur in a kind of virtual workspace. The role of $\mathrm{HOC}$, from an evolutionary point of view, may indeed be to provide a degree of resilience in the face of potential structural damage to the neural architecture [1]. HOC has the capacity to rearrange processes within the brain and assure "business as usual" notwithstanding adverse conditions. Thinking about actions in terms of thoughts and feelings (mentalizing), using knowledge of these mental states to master life challenges (metacognitive mastery, or the ability to respond to psychological challenges effectively on 
Adversity, attachment, and mentalizing

the basis of psychological knowledge $[6,9]$ ) or designating them as "just thoughts" that do not need to be acted on (in the context of mindfulness) or that, by contrast, need to be seriously and maturely considered (for the purpose of reflection, as in RF) all serve this purpose of creating a higher-order process above cognition (including cognition regarding emotions).

We describe this conceptualization in order to signal that we are not going to be concerned in this discussion about conceptual and measurement differences between those who work with the notion of metacognition or mentalizing or social cognition or RF. The authors of the papers in this special issue assiduously define the concept they use and describe the measurement approach they have created in relation to it. But, in our view, while they are no doubt necessary to create scientific discourse, these definitional issues are largely irrelevant at the current state of knowledge. They all represent approximations, each highlighting some unique features but all attempting to strive towards explanations of personality disorder where some dysfunctional characteristics of a higher-order cognitive system may be seen to account for personality disorder. We will use the term mentalizing for the purpose of this commentary in a generic sense which will cover metacognitive, mindful or reflective self-awareness. ${ }^{1}$

The HOC model of personality disorder and its relationship to adversity and attachment has been presented from a range of perspectives in the past, and most of these find echoes in the present collection of intriguing reports. Here we would like to highlight five of these. First, one manifestation of borderline personality disorder (BPD) is poor mentalizing, with different problems arising at different stages of the disorder, dependent on the mentalizing profile of the individual $[4,10,11]$.

\footnotetext{
${ }^{1}$ This is not an imperialistic attempt to conquer scientific territory and "assert the supremacy" of mentalizing over other constructs. It is simply that we know what we mean by the term. We may be less sure-footed in using the language of metacognition or mindfulness.
} 
Adversity, attachment, and mentalizing

Second, the attachment system in BPD may be disordered, and the problems of the attachment system may create a vulnerability in relation to interpersonal interaction and intimate relationships [12], with ultimate responsibility for such disruptions attributed to the inflexibility of processing systems governing social (relationship) expectations [13].

Third, there is suggestive evidence that mentalizing can protect from the expression of symptoms and it is the failure of that protection that makes someone symptomatic $[14,15]$.

Fourth, developmentally, the quality of attachment and mentalizing interact in complex causal ways: while a benign attachment context is considered to enhance mentalizing, the experience of trauma, and understanding and overcoming adversity, could also be considered to entail the enhancement of mentalizing in the process of overcoming trauma [16].

Fifth, from a measurement standpoint, there may be significant confounding factors. Self-reports or perceptions of attachment relationships are likely to be conditioned by mentalizing - a better-mentalized picture of a relationship is likely to be balanced and is less likely to be unequivocally positive or negative $[17,18]$. Thus, there is a built-in correlation between some measures of mentalizing and some measures of attachment. The present authors are as guilty of failing to draw the reader's attention to such item overlap as any of the other contributors. In terms of the current commentary, we simply need to sound a cautionary note about the inherent interdependence of the attachment and mentalizing systems as well as attachment and affect regulation at the measurement level. In other words, measured attachment may well depend on the quality of mentalizing and the quality of affect regulation. These systems are loosely coupled, and their truly independent measurement may be both conceptually and practically artificial. 
Adversity, attachment, and mentalizing

\section{Substantive discussion}

The paper by Antonsen et al. [19] (this issue) reports on the association of RF based on adult attachment interviews, clinical parameters and treatment outcome for people with personality disorder. The population studied comprised patients who participated in a randomized trial comparing a combined short intensive day hospital stabilization phase followed by individual and group psychotherapy to outpatient psychotherapy. RF was measured on entry to the study and its relationship with long-term outcomes assessed. On the whole, as might be expected, symptom severity, interpersonal problems, wellbeing, self-esteem, identity integration and relational capacities all favour the group of patients with personality disorder who have relatively high RF. However, not all results were consistent with mentalizing theory. Two core indicators, the Global Assessment of Functioning (GAF) (generalized adaptation) and the self-control domain of the Severity Indices of Personality Problems (SIPP-118), did not relate to RF. Mentalizing theory predicts a relationship of RF with both adaptation and selfcontrol [20].

What might account for this selective failure of replication? As all the patients were in the trial because of poor adaptation and self-control problems, it is possible that the range of variance on these measures was too small to show strong associations; in essence, these studies ran into a problem of floor effects on these measures. Although this possibility is raised by the authors, given the robustness of the instruments concerned, we consider it unlikely. It is more likely that the point raised above about the interactive nature of measurement systems in this area came into play. It is likely that individuals with medium RF have different perceptions and standards of reporting self-control than those with low RF. It is axiomatic that RF should influence the perception of self-control since RF is an indicator of its quality. Those struggling to represent their psychological experiences will find it challenging to accurately identify when they are not in control. Therefore, on occasions, they 
Adversity, attachment, and mentalizing

will erroneously judge themselves to be in control when in reality they are not. Those with higher RF may, intriguingly, be more aware of self-control problems and therefore report it as relatively more severe and more prominent. One may imagine a similar process at play in relation to GAF score results. To the extent that GAF scores are relatively subjective evaluations by clinicians, it is possible that patients with higher RF showed greater awareness of their struggles with adaptation, which were then "objectively" rated as more severe.

$\mathrm{RF}$ as a moderator of outcome shows, at first sight, some paradoxical results. Patients with BPD or avoidant personality disorder with medium RF did better in more intensive treatment, while those with lower RF improved more with lower-intensity outpatient treatment. The authors mention at least two categories of explanation for this observation. First, we know that "the rich get richer"; to those that have more, more shall be given. What we mean by this is that individuals with high RF can gain more out of any social situation because they have a deeper understanding of interpersonal processes. Second, day hospital treatment, while less intensive than inpatient treatment, stimulates interpersonal interaction, the core of personality pathology, more than community-based outpatient treatment. In other words, intensive treatments have greater potential to increase the underlying pathology in individuals whose attachment system is hypersensitive. In essence, combining the two accounts, a moderate or high level of RF allows a patient to take advantage of the beneficial aspects of any treatment while protecting themselves from the harmful effects of overarousal. For example, a more reflective patient is more likely to be able to ignore clinician errors and the potential iatrogenic effects of critical responses of other patients in the treatment programme. This reflects the resilience-promoting aspect of mentalization; higher RF enables them to overcome daily challenges as well as more dramatic adversity. We have previously commented that social stimulation in day hospital treatment and outpatient combined individual and group treatment may be too high for individuals with personality 
Adversity, attachment, and mentalizing

disorder [21], particularly so for patients with antisocial personality disorder (ASPD) [22]. It is not solely attachment processes that disrupt mentalizing; group inclusion and anxiety about social rank may also undermine mentalizing [7, 23]. Patients with personality disorders often manage their own level of contact in intensive treatment, and we may predict that this may be evidenced in attendance and drop-out rates. Drop-out could be a deprecatory misnomer in this context as, in effect, we are referring to people protecting themselves from potentially iatrogenic effects of therapy. While the drop-out rate was higher in the combined treatment in the original trial on which this study is based, the difference was not statistically significant, and the differences in attendance rate were unclear.

Finally, mentalizing theory suggests that there should be predictive value of RF for long-term clinical outcome of treatment [24], which is not observed in this study. Is this inconsistent with the theory? Possibly so, but individuals with low RF were found to be more impaired in this study on the main outcome measures. Consequently, low RF individuals had more room to improve, while people with higher RF had more capacity to improve because of their higher RF. The result of no difference may be overturned if controlling for initial values shows a correlation with outcome.

The preliminary study by Brüne et al [25] (this issue) uses well-matched but small samples. Unfortunately, the groups could not be matched for history of childhood trauma. While the BPD group reported high levels of childhood trauma and low mentalizing, which is to be expected, some caution may be required in interpreting the findings because low mentalizing may be significant in explaining the toxic effects of trauma on personality functioning. Furthermore, self-report measures of childhood adversity were used, and people who are distressed are more likely to recall negative life experiences, providing a built-in correlation between disorder severity and trauma [26]. 
Adversity, attachment, and mentalizing

The study represents a substantial contribution because it introduced a new task with the potential to help clarify the contradictory results in relation to mentalizing and BPD, which some reviews of the literature have reported. Standard tasks of mentalizing fail to tap into the complex emotions relating to sexuality, rejection and lack of trust that patients with a diagnosis of BPD tend to bring. Patients with BPD were found to score more poorly on this task regardless of whether the task tapped cognitive or emotional mentalizing. The task was robust to symptom severity. However, both cognitive and affective mentalizing correlated with neglect and both physical and sexual abuse. There are two results to explain here. One concerns the nature of the association of both cognitive and affective mentalizing with BPD, where, notably, affective mentalizing was thought to be intact [27], and the second concerns the interrelationship of attachment styles, mentalizing and childhood trauma.

Normally, cognitions and emotions are balanced against each other, so when there is a lack of cognitive moderation of emotional responses mentalizing problems become apparent. In BPD, mentalizing theory suggests that any apparent reduction in cognitive mentalizing is not simply a deficit in cognitive mentalizing but also the consequence of the dominance of emotion-dominated processing [28]. People with BPD may at times lack the capacity to contextualize emotional experiences through cognitive coherence; this is what creates the problem. Emotions are unmoderated by contextualization because cognitions about affect are limited and patients cannot think about emotions. The excessive emotional reactivity in BPD, which results in an apparent cognitive deficit, may arise because sensitivity to emotional cues is greater than in individuals who do not have BPD $[29,30]$ when affect is unmoderated by cognition. This model would predict that both cognitive and affective mentalizing would be hampered.

Interestingly, in Brüne et al.'s study [25] attachment insecurity, as indicated by a continuous measure of dismissing attachment style, was not associated with BPD features, 
Adversity, attachment, and mentalizing

but this could simply reflect low power and be related to sample size. By contrast, preoccupied attachment style predicted the greatest disruption to mentalizing. This confirms earlier studies in which attachment style has been linked to limitations in mentalizing [31]. However, it may be too simplistic to accept that preoccupied attachment style itself is the cause of mentalizing problems. Preoccupied attachment may be a manifestation of problems with cognitive forms of mentalizing. Preoccupation as a state of mind in relation to attachment experiences [32] arguably involves sacrificing certain more balanced and reflective ways of thinking about mental states. So it may be inappropriate to place attachment style and mentalizing in a causal sequence here - they may be describing the same phenomena from different starting points. Furthermore, more sophisticated statistical modelling may be necessary to get a more accurate picture of the interrelationships. In this study, the sample was not large enough to construct a mediational model for exploring how the relationship of early neglect and mentalizing may have been moderated or mediated by, for example, attachment style. The authors used multiple linear regressions and found that neglect, particularly physical neglect, shared most variability, and represented the other adversity variables.

Setting aside the lack of comparability of the two groups in terms of trauma experience, further comparison would have been possible given a larger sample size, perhaps to identify that BPD arises not so much as a consequence of trauma but more a result of not being resilient to trauma [Fonagy, 2014 \#6583;Fonagy, 2015 \#7178]. Such findings could be interpreted as suggesting that mentalizing contributes to a process of resilience, which is what could be reflected by the findings of mentalizing problems, attachment and trauma diagnoses reported by Brüne et al. [25]. The fact that the control sample in this study was taken from a non-clinical population leaves us with the intriguing possibility that the elevated cognitive and affective empathy measured in the control group has helped to protect the individuals 
Adversity, attachment, and mentalizing

concerned from some of the negative mental health outcomes associated with experiencing adversity in childhood.

Another study in this issue looking at attachment and mentalizing quantified the latter as metacognitive mastery (knowledge about mental states of self and others) and attachment using a self-report measure. The paper, by Outcalt et al. [33] (this issue), links anxious attachment to BPD via low metacognition in a sample of individuals with substance abuse problems. With a sample of mostly male patients with BPD traits receiving residential treatment for substance abuse, the study looked at the interaction of metacognition, attachment, and BPD traits. Metacognitive mastery moderated anxious attachment so that the combination of poor metacognition and anxious attachment predicted BPD traits. In general, both mastery and anxious attachment were less with increasing numbers of BPD features. With an interaction term combining mastery and anxious attachment, the prediction of BPD features improved. As the authors point out, the findings not only have value in pointing clinicians towards a more precise description of the experience of people with BPD, but also suggest that some aspects of metacognitive mastery can be used in clinical interventions, perhaps most usefully in substance abusing clinical group.

Can we be equable about mastery as a moderator that protects individuals from or exposes them to the impact of anxious attachment? As we stressed above, treating attachment and metacognition as independent and only obliquely connected phenomena may be problematic; they may not be as self-evidently separable or independent as they are implicitly considered in this moderational analysis. Attachment is a loosely coupled construct with HOC, and so combining them into a single model that assumes independence is questionable. We appreciate that this was necessary and is of interest, but nevertheless it may be flawed conceptually. The authors suggest that when metacognition is low, anxious attachment can "cause" BPD. An oversimplified interpretation of this suggestion is that attachment 
Adversity, attachment, and mentalizing

experiences associated with anxious attachment, if moderated by metacognitive mastery, do not increase vulnerability to BPD. Our interpretation of this is that subjective experiences linked to anxious attachment history create internal disorganization and metacognition moderates the impact. If the disorganization is not managed through metacognition, this leads to behaviours characteristic of BPD, such as self-harm, because of the re-emergence of nonmentalizing modes.

Anxious attachment may cause disturbed relationship expectations, formulated in clinical practice as anxious attachment style, but not symptoms of BPD such as self-harm. Overall, there should be a correlation between metacognition and BPD given that many BPD symptoms are direct indications of metacognitive problems, for example, impulsivity or affect dysregulation. Perhaps it is the inability to process disorganized feelings and ideas of attachment that causes the problem. If an individual has secure attachment and becomes stressed, then this stress will not lead to symptoms. Presumably if an individual has high dismissing scores they are withdrawing from social contact and so there is less need for metacognitive mastery. More research is needed.

The study by Petersen et al. [34] (this issue), in which the researchers assess the cognitive and affective mentalizing ability of patients with BPD compared with matched controls by using a range of well-established Theory of Mind tests assessing simple and complex mentalizing tasks, shows few anomalies in BPD on mentalizing ability in simple mentalizing tasks but increasing problems with more complex tasks involving humour or understanding others' motives, reflecting the findings reported by Brüne et al. [25]. This is consistent with previous studies that show that differences emerge when additional load is placed on cognitive and emotional processing and when tasks represent higher interpersonal demands. Differences were modest but in the expected direction. Importantly, the authors used standardized tasks frequently used by other researchers, which opens the door to future 
Adversity, attachment, and mentalizing

studies. For example, it would be of interest to map the developmental emergence of these mentalizing anomalies from pre-adolescence onwards. Faux pas tasks can be completed from 6 years onwards, so it would be informative to find out when these problems emerge and if they predict emergence of BPD. We already have indications that Theory of Mind problems at age 4 or 5 may predict BPD in adolescence [35], and hypermentalizing in adolescents with BPD also predicts poorer outcomes [11,36].

Petersen et al. [34] also investigated whether there was a relationship between current mentalizing and childhood adversity. They found that as reports of childhood punishment from parents increased, mentalizing was increasingly undermined. While we may speculate, ultimately the mechanism for this remains obscure. It may be a mechanism that is associated with poorer mentalizing following trauma: trauma weakens mentalizing and this results in a loss of resilience. This places an individual at risk of symptoms of various mental disorders. Certainly, all the papers in this special issue point towards the need for further understanding of how trauma is processed. We suggest that the adverse effects of trauma may operate through some form of phobic avoidance of the threat that mental states begin to imply to the child. To engage in the thoughts and feelings of others exposes these children to frankly malevolent mental states from parents or other adults. It could be safer for the child not to engage in a punishing social network. There is evidence to suggest that the quality of maternal mentalizing in mothers of sexually abused children is reduced when compared with matched controls [37]. However, it is the social network that promotes the development of mentalizing, and therefore trauma, by prompting a psychological withdrawal, may cause their mentalizing to become compromised. Alternatively, the same phenomena could be explained arguably more parsimoniously as secondary to anxiety and dysregulated affect: punishment increases anxiety and mentalizing suffers as a consequence [38]. 
Adversity, attachment, and mentalizing

Velotti et al. [39] report a preliminary study on the relationship between mindfulness, aggression and ASPD traits based in a prison population. The authors consider a mindfulness measure to be an index of mentalizing. The extent to which mentalizing and mindfulness are overlapping or distinct constructs may be unclear, but from the point of view of this commentary both are considered to be HOCs. While the content of measures indicate a high level of overlap [4], interventions to enhance mentalizing, for example, in mentalizationbased treatment [40], and mindfulness, for example, in dialectical behaviour therapy and metacognitive conceptualizations and treatment of personality disorders [41, 42], are somewhat different. Despite differences in therapeutic orientation, the mechanisms of action of these treatments may have much in common. This is an intriguing question, which is the focus of much current concern in the field [43]. Velotti et al. report that, unsurprisingly, mindfulness is lower when levels of overt aggression are high. Similarly, whether ASPD is assessed continuously in terms of number of features or dichotomously as a diagnosis, it is associated with lower levels of mindfulness. Low mindfulness seems to be a sufficient indicator for the diagnosis of ASPD in this sample, as does high aggression. High aggression and high mindful individuals may be high on psychopathy levels. The study suggests that individuals who are low on aggression manifest a diagnosis of ASPD only if they are also low on mindfulness; low mindfulness is associated with ASPD traits even in the absence of aggression. So, an individual can become antisocial without aggression if their capacity to mentalize is limited. It seems that if someone is able to mentalize to a reasonable level it is possible to work around other risk factors for antisocial behaviour. By contrast, in the presence of aggressive behaviour, the degree of mindfulness (mentalizing) matters little, or at least less. These conclusions sound very definitive. However, the sample size of the study is modest, and replications of these important associations are urgently required. 
Adversity, attachment, and mentalizing

Sharp et al. [44] (this issue) report a meditational model where attachment drives the anomaly of mentalizing, which in turn predicts BPD symptoms. This is a large study based on a clinical sample of consecutive admissions to an inpatient adolescent unit who were tested on a sophisticated interview-based measure of attachment, the Child Attachment Interview [45]. Arguably, interview-based measures of attachment are preferable to selfreport instruments [46]. The great strengths of Sharp et al.'s study are that it does not confound BPD as a syndrome with clinical status, and it controls for the level of distress. Furthermore, the paper contrasts a mentalizing [47] and an affect dysregulation [48] model of BPD. The authors contrast affect regulation with hypermentalizing as two potential mediators of attachment, and find that hypermentalizing and not emotional dysregulation mediates the link between attachment coherence and borderline features. This result is consistent with Sharp et al.'s compelling model of adolescent BPD [11]. It is also consistent with accumulating evidence that hypermentalizing (at least at this age) is uniquely linked to BPD symptoms, including to symptoms of low cognitive empathy [49]. Hypermentalizing may not be a significant predictor of either cognitive or emotional empathy in other populations where affect regulation probably plays a larger role across a number of social cognitive domains [49].

The evidence for hypermentalizing in patients with BPD traits is now well replicated, but we do not yet know whether older patients with BPD manifest similar characteristics. Furthermore, evidence is accumulating in relation to affect dysregulation dominating the clinical picture of adults with BPD. Some may argue that hypermentalizing is but a precursor to full-blown BPD, only to be found among adolescents. Momentary ecological assessment studies are attempting to unpick the interaction between impulsivity and the social context $[50,51]$. When measures are taken on multiple occasions during a day, the time series models that can be fitted to the data have the potential to reveal the sequence, if not yet the causal 
Adversity, attachment, and mentalizing

relationship between subjective states. We know that expressed affect as it is remembered bears little relationship to affect as recorded [52], with a high likelihood that impulsivity seems to be so in name alone. BPD patients build up to impulsive acts over time. It would be intriguing to monitor the relationship of ongoing mentalizing capacity, emotion dysregulation and "impulsive" difficulties such as suicidality. Unfortunately, we do not yet have momentary measures of hypermentalizing that could establish the cross-sectional causal relationships depicted in Sharp et al.'s paper unfolding in real time. It is perhaps not inappropriate to conclude that even this excellent paper leaves us with the need for more research.

\section{Conclusion of the discussion}

To conclude, the majority of papers in this special issue deal with the relationship of adversity, attachment, mentalizing and borderline symptoms. It is gratifying that many, if not most, of the findings we have reviewed are consistent with the model proposed by Fonagy and Luyten [28]. That model distinguishes distal and proximal causes for the genesis of symptoms of BPD and hypothesizes interactions between components at each of these levels. Distal factors include constitutional causes not considered by the papers in this issue and the context and process of early caregiving and maltreatment considered by Brüne et al. [25], Outcalt et al. [33], Sharp et al. [44] and Petersen et al. [34] in this issue. More proximal causes involve sensitivity to stress and arousal, in particular but not limited to attachmentrelated arousal. Variables in this category were considered by Velotti et al. [39], Sharp et al. [44] and Petersen et al. [34]. The model suggests that distal factors lead to disruptions of the attachment system, which in turn generate problems of self-other differentiation because activation of the attachment system frequently brings with it mentalizing failure [53]. 
Adversity, attachment, and mentalizing

On the whole, the studies support both the link between adversity and mentalizing failure [e.g., 25] and non-secure attachment and poor mentalizing [33, 44]. The model suggested above goes beyond the data from the studies included in this special issue. It suggests that problems in maintaining an equilibrium between responding with resonance to the other, which empathy requires, and maintaining a reflective separateness to appreciate the independence of self from others' perspectives generate difficulties in mentalizing. The intriguing findings of the treatment study of Antonsen et al. [19], which showed patients with high RF to benefit more from intensive treatment, is consistent with this kind of dynamic model, where the vulnerability of mentalizing threatens the sense of identity in close interpersonal situations. A hypersensitivity to mental states of both self and other (hypermentalizing) emerges, together with a low threshold for activation of attachment and co-occurring deactivation of mentalizing. Velotti et al.'s [39] findings of aggression fit neatly with this model. The overall picture one can build from these studies is one of the progressive impairment of integration of cognitive and affective processes and the fading of robust selfother differentiation in severe personality disorder.

In essence, vulnerable mentalizing (hypermentalizing) triggers attachment disorganization, which in turn triggers arousal, which in turn undermines further mentalizing. It is this mechanism, which is seen as acquired, that brings proximal causes to the forefront, triggering the characteristic phenomenological and behavioural symptoms of BPD that are themselves prementalizing modes of subjectivity, described some years ago as psychic equivalence, pretend mode and teleological function [54]. By and large, the studies reported in this issue have assumed, rather than demonstrated, how low mentalizing brings forth forms of cognitive function that developmentally antedate "thinking about thinking" $[55,56]$. In fact, the literature contains only qualitative studies that fully describe the subjective experience of individuals with BPD - that is, what nonmentalizing feels like from within. 
Adversity, attachment, and mentalizing

Largely this is a methodological issue, but it is also a conceptual one. It is obviously challenging to study, using primarily the tools of symbolic representation (e.g., language), a set of phenomenological experiences that are probably characterized by being essentially beyond words. We often refer to the intensity of emotional experience in BPD, but struggle to quantify it because by the time the reflective mind that could participate in such a quantification comes on stream, the phenomenon of interest is no longer there. Nevertheless, the findings reported in papers in this special issue are broadly consistent with this model, although, of course, they might also confirm alternative models - but that is not in our brief to explore.

There are also inconsistencies. It is, for example, suggested that adversity and/or attachment models may have an impact on personality disorder in the absence of problems with metacognition [39], but there also seems to be evidence that the effects of adversity are mediated through changes in metacognition/mentalizing [33]. Some of the studies have modest sample sizes, making these complex interactional relationships difficult to establish with certainty, so clearly further studies are needed. There are some inconsistencies between the findings, for example, in relation to mentalizing as a moderator [33] or as a consequence [25] of early adversity. These differences may, in part, be due to the different studies measuring these constructs somewhat differently. When talking of mentalizing, metacognition, social cognition, mindfulness and RF, we appear to be addressing a similar or at least overlapping set of higher cognitive capacities. Yet the diversity of operationalization of this fundamental construct remains a major challenge for the field. The lack of standard operationalization highlights a more serious problem, namely, that researchers often do not start with a developmental model of how mental health symptoms, attachment, adversity and mentalizing normally interrelate across development, and then test such models in longitudinal investigations. Work on treatment moderation comes closest to this, but such 
Adversity, attachment, and mentalizing

developments will be piecemeal and based on associations observed in small trials [19]. Overall, what is required are large-scale studies using an agreed model that can be tested using robust measures $[57,58]$ rather than trying to piece together a model from disparate studies.

This leaves us with the issue of the heterogeneity of the HOC construct included in this issue of the journal. There is evidently more work needed to help clarify the relationship between overlapping ideas such as metacognition and mentalizing. We could have spent this entire discussion addressing the similarities and differences of these ideas and measurement systems, but we viewed such an exercise as probably unproductive at their current state of psychometric and conceptual evolution. Discussions of that sort run the serious risk of reifying and ossifying ideas that are best left to develop naturally in the hurly-burly and rough and tumble of "everyday science" [59]. We think that comparative research will ultimately be required to align these concepts, which are currently helpfully used in specific clinical contexts. At the present state of our knowledge, premature attempts at arriving at an integrated model may bring "pseudo-exactitude" and speculation. Ultimately, empirical and conceptual distinctions between perspectives on HOC will need to be addressed in large-scale studies. In the absence of large-scale investigations, there is a risk that we will begin to describe an elephant by focusing on one specific aspect and fail to see the whole.

\section{References}

1. Rudrauf D. Structure-function relationships behind the phenomenon of cognitive resilience in neurology: insights for neuroscience and medicine. Adv Neurosci 2014;2014:1-28.

2. Mante V, Sussillo D, Shenoy KV, Newsome WT. Context-dependent computation by recurrent dynamics in prefrontal cortex. Nature 2013;503:78-84. 
Adversity, attachment, and mentalizing

3. Chiesa M, Fonagy P. Reflective function as a mediator between childhood adversity, personality disorder and symptom distress. Personality and Mental Health 2014;8:52-66.

4. Luyten P, Fonagy P, Lowyck B, Vermote R. Assessment of mentalization. In: Bateman AW, Fonagy P, editors. Handbook of mentalizing in mental health practice. Washington, DC: American Psychiatric Press; 2012. p. 43-66.

5. Sharp C, Fonagy P, Goodyer I. Social cognition and developmental psychopathology. Oxford, UK: Oxford University Press; 2008.

6. Semerari A, Carcione A, Dimaggio G, Falcone M, Nicolò G, Procacci M, et al. How to evaluate metacognitive functioning in psychotherapy? The metacognition assessment scale and its applications. Clin Psychol Psychother 2003;10:238-61.

7. Dimaggio G, Lysaker PH. Metacognition and mentalizing in the psychotherapy of patients with psychosis and personality disorders. J Clin Psychol 2015;71:117-24.

8. Brown KW, Ryan RM. The benefits of being present: mindfulness and its role in psychological well-being. J Pers Soc Psychol 2003;84:822-48.

9. Carcione A, Nicolo G, Pedone R, Popolo R, Conti L, Fiore D, et al. Metacognitive mastery dysfunctions in personality disorder psychotherapy. Psychiatry Res 2011;190:60-71.

10. Ensink K, Normandin L, Target M, Fonagy P, Sabourin S, Berthelot N. Mentalization in children and mothers in the context of trauma: An initial study of the validity of the Child Reflective Functioning Scale. Br J Dev Psychol 2015;33:203-17.

11. Sharp C, Ha C, Carbone C, Kim S, Perry K, Williams L, et al. Hypermentalizing in adolescent inpatients: treatment effects and association with borderline traits. J Pers Disord 2013;27:3-18.

12. Gunderson JG, Lyons-Ruth K. BPD's interpersonal hypersensitivity phenotype: a geneenvironment-developmental model. J Pers Disord 2008;22:22-41. 
Adversity, attachment, and mentalizing

13. Deckers JW, Lobbestael J, van Wingen GA, Kessels RP, Arntz A, Egger JI. The influence of stress on social cognition in patients with borderline personality disorder. Psychoneuroendocrinology 2015;52:119-29.

14. Fonagy P, Steele M, Steele H, Higgitt A, Target M. The Emanuel Miller Memorial Lecture 1992. The theory and practice of resilience. J Child Psychol Psychiatry 1994;35:23157.

15. Berthelot N, Ensink K, Bernazzani O, Normandin L, Luyten P, Fonagy P. Intergenerational transmission of attachment in abused and neglected mothers: the role of trauma-specific reflective functioning. Infant Ment Health J 2015;36:200-12.

16. Greenberg DM, Baron-Cohen S, Rosenberg N, Fonagy P, Rentfrow PJ. Elevated empathy in adults with childhood trauma. Manuscript submitted for publication. 2015.

17. Ensink K. Assessing theory of mind, affective understanding and reflective functioning in primary school aged children [PhD thesis]. London, UK: University of London; 2003. 18. Target M, Shmueli-Goetz Y, Fonagy P, Datta A. Attachment representations in schoolage children: The development and validity of the Child Attachment Interview (CAI). Unpublished manuscript. London, UK: University College London; 2006. 19. Antonsen BT, Johansen MS, Ro FG, Kvarstein EH, Wilberg T. Is reflective functioning associated with clinical symptoms and long-term course in patients with personality disorders? Compr Psychiatry 2015.

20. Fonagy P, Gergely G, Jurist E, Target M. Affect regulation, mentalization, and the development of the self. New York, NY: Other Press; 2002.

21. Fonagy $\mathrm{P}$, Bateman A. Progress in the treatment of borderline personality disorder. $\mathrm{Br} \mathbf{J}$ Psychiatry 2006;188:1-3.

22. Bateman A, Bolton R, Fonagy P. Antisocial personality disorder: A mentalizing framework. FOCUS 2013;11:178-86. 
Adversity, attachment, and mentalizing

23. Liotti G, Gilbert P. Mentalizing, motivation, and social mentalities: theoretical considerations and implications for psychotherapy. Psychol Psychother 2011;84:9-25; discussion 98-110.

24. Fonagy P, Leigh T, Steele M, Steele H, Kennedy R, Mattoon G, et al. The relation of attachment status, psychiatric classification, and response to psychotherapy. J Consult Clin Psychol 1996;64:22-31.

25. Brüne M, Walden S, Edel MA, Dimaggio G. Mentalization of complex emotions in borderline personality disorder: The impact of parenting and exposure to trauma on the performance in a novel cartoon-based task. Compr Psychiatry 2015.

26. Watkins E, Teasdale JD. Adaptive and maladaptive self-focus in depression. J Affect Disord 2004;82:1-8.

27. Harari H, Shamay-Tsoory SG, Ravid M, Levkovitz Y. Double dissociation between cognitive and affective empathy in borderline personality disorder. Psychiatry Res 2010; 175:277-9.

28. Fonagy P, Luyten P. A developmental, mentalization-based approach to the understanding and treatment of borderline personality disorder. Dev Psychopathol 2009;21:1355-81.

29. Lynch TR, Rosenthal MZ, Kosson DS, Cheavens JS, Lejuez CW, Blair RJ. Heightened sensitivity to facial expressions of emotion in borderline personality disorder. Emotion 2006;6:647-55.

30. Franzen N, Hagenhoff M, Baer N, Schmidt A, Mier D, Sammer G, et al. Superior 'theory of mind' in borderline personality disorder: an analysis of interaction behavior in a virtual trust game. Psychiatry Res 2011;187:224-33. 
Adversity, attachment, and mentalizing

31. Sharp C, Fonagy P. The parent's capacity to treat the child as a psychological agent: Constructs, measures and implications for developmental psychopathology. Soc Dev 2008; 17:737-54.

32. Main M, Hesse E, Goldwyn R. Studying differences in language usage in recounting attachment history: an introduction to the AAI. In: Steele H, Steele M, editors. Clinical applications of the Adult Attachment Interview. New York, NY: Guilford Press; 2008. 33. Outcalt J, Dimaggio G, Popolo R, Buck K, Chaudoin- Patzoldt KA, Olesek KA, et al. Metacognition moderates the relationship of disturbances in attachment with severity of borderline personality disorder among persons in treatment for substance use disorders. Compr Psychiatry 2015.

34. Petersen R, Brakoulias V, Langdon R. An experimental investigation of mentalization ability in borderline personality disorder. Compr Psychiatry 2015.

35. Belsky DW, Caspi A, Arseneault L, Bleidorn W, Fonagy P, Goodman M, et al. Etiological features of borderline personality related characteristics in a birth cohort of 12year-old children. Dev Psychopathol 2012;24:251-65.

36. Sharp C, Pane H, Ha C, Venta A, Patel AB, Fonagy P. Theory of mind and emotion regulation difficulties in adolescents with borderline traits. J Am Acad Child Adolesc Psychiatry 2011;50:563-73.

37. Ensink K, Normandin L, Leroux A, Biberdzic M, Fonagy P. Assessing reflective parenting in interaction with school-aged children. J Pers Assess. (Submitted for publication). 2015.

38. Kruger A, Ehring T, Priebe K, Dyer AS, Steil R, Bohus M. Sudden losses and sudden gains during a DBT-PTSD treatment for posttraumatic stress disorder following childhood sexual abuse. Eur J Psychotraumatol 2014;5:doi: 10.3402/ejpt.v5.24470. 
Adversity, attachment, and mentalizing

39. Velotti P, Garofalo C, D'Aguanno M, Petrocchi C, Popolo R, Salvatore G, et al. Mindfulness moderates the relationship between aggression and antisocial personality disorder traits: preliminary investigation with an offender sample. Compr Psychiatry 2015. 40. Bateman AW, Fonagy P. Mentalization-based treatment for personality disorders: A practical guide. Oxford, UK: Oxford University Press; 2016 (in press).

41. Linehan MM, Wilks CR. The Course and Evolution of Dialectical Behavior Therapy. Am J Psychother 2015;69:97-110.

42. Ottavi P, Passarella T, Pasinetti M, Salvatore G, Dimaggio G. Adapting mindfulness for treating personality disorder. In: Livesley WJ, Dimaggio G, Clarkin JF, editors. Integrated treatment for personality disorder: A modular approach. New York, NY: Guilford Press; 2015. p. 282-303.

43. Links PS. Developing personalised integrated psychotherapy for patients with personality disorders. Evid Based Ment Health 2015;18:22.

44. Sharp C, Venta A, Vanwoerden S, Schramm A, Ha C, Newlin E, et al. First empirical evaluation of the link between attachment, social cognition and borderline features in adolescents. Compr Psychiatry 2015.

45. Shmueli-Goetz Y, Target M, Fonagy P, Datta A. The Child Attachment Interview: a psychometric study of reliability and discriminant validity. Dev Psychol 2008;44:939-56. 46. Fonagy P, Stein H, Jacobs N, Ferguson K, Allen J. What do adult attachment scales measure? Bull Menninger Clin 1998:33-82.

47. Bateman A, Fonagy P. Mentalization based treatment for borderline personality disorder. World Psychiatry 2010;9:11-5.

48. Crowell SE, Beauchaine TP, Linehan MM. A biosocial developmental model of borderline personality: elaborating and extending Linehan's theory. Psychol Bull 2009;135:495-510. 
Adversity, attachment, and mentalizing

49. Kalpakci A, Vanwoerden S, Elhai JD, Sharp C. The independent contributions of emotion dysregulation and hypermentalization to the "double dissociation" of affective and cognitive empathy in female adolescent inpatients with BPD. J Pers Disord 2015:doi:

10.1521/pedi_2015_29_192.

50. Tomko RL, Brown WC, Tragesser SL, Wood PK, Mehl MR, Trull TJ. Social context of anger in borderline personality disorder and depressive disorders: findings from a naturalistic observation study. J Pers Disord 2014;28:434-48.

51. Tomko RL, Solhan MB, Carpenter RW, Brown WC, Jahng S, Wood PK, et al. Measuring impulsivity in daily life: the momentary impulsivity scale. Psychol Assess 2014;26:339-49. 52. Brown WC, Tragesser SL, Tomko RL, Mehl MR, Trull TJ. Recall of expressed affect during naturalistically observed interpersonal events in those with borderline personality disorder or depressive disorder. Assessment 2014;21:73-81.

53. Nolte T, Bolling DZ, Hudac CM, Fonagy P, Mayes L, Pelphrey KA. Brain mechanisms underlying the impact of attachment-related stress on social cognition. Front Hum Neurosci $2013 ; 7: 816$.

54. Fonagy P. An attachment theory approach to treatment of the difficult patient. Bull Menninger Clin 1998;62:147-69.

55. Fonagy P, Target M. Playing with reality: III. The persistence of dual psychic reality in borderline patients. Int J Psychoanal 2000;81:853-73.

56. Fonagy P, Target M. Playing with reality: IV. A theory of external reality rooted in intersubjectivity. Int J Psychoanal 2007;88:917-37.

57. Fonagy P, Allison E. The role of mentalizing and epistemic trust in the therapeutic relationship. Psychotherapy 2014;51:372-80. 
Adversity, attachment, and mentalizing

58. Fonagy P, Luyten P, Allison E. Epistemic petrification and the restoration of epistemic trust: a new conceptualization of borderline personality disorder and its psychosocial treatment. J Pers Disord 2015;29:575-609.

59. Kuhn TS. The structure of scientific revolutions. Chicago: University of Chicago Press; 1962. 